\title{
Availability of Stochastic Degrading Systems Subject to Imperfect Repair
}

\author{
Jiang Yun Chun*, Xiang You Huan* and Chen Xi Xiang
}

Technical Department, Taiyuan Satellite Launch Center, Taiyuan 030027, China

\begin{abstract}
A series repairable system not only is one of the classical reliability models but is also the usually used model in practice. In this paper, a method for calculating system availability for series repairable system is studied. From the states of components, this methodology uses a gamma distribution to model the material degradation, and the impact of imperfect maintenance actions on the system reliability is investigated. The state of a degrading system immediately after the imperfect maintenance action is assumed as a random variable and the maintenance time follows a geometric process. At last, a numerical example is presented to demonstrate the use of the model.
\end{abstract}

Keywords: Availability, degrading systems, imperfect repair, renewal process, system engineering.

\section{INTRODUCTION}

Availability, which is the probability that the system is up at any time has been considered as an important index in the defense and aerospace industry. In the earliest study of the system availability problem, the repair-replacement models mainly concentrate on the study of perfect repair models in which the system after repair is as good as new. In practice, most systems deteriorate due to ageing effects and accumulated wear. In other words, a system after repair cannot be as good as new. An imperfect repair model in which a repair with probability $p$ is a perfect repair, and with probability $q=1-p$ is a minimal repair, was first introduced by Brown and Proschan [1]. Pham and Wang [2] provided a thorough survey and review of the eight imperfect maintenance models. For a deteriorating simple repairable system, its successive working times of the system after repair may become shorter and shorter while the consecutive repair times of the system may become longer and longer. To model such a deteriorating system, Lam [3] first introduced a geometric process repair model. Other works on the geometric process model in maintenance analysis include Lam [4], Zhang [5] and Wang [6], et al. Many research works have been done by Kijima [7], Martorell [8], Sheu [9], Chiang \& Yuan [10], Jiang \& Ji [11], and others along this direction of imperfect repair model.

Lyer [12] investigated the availability model based on $(p, q)$ policy. Zhao [13] founded the availability model of series repairable systems using alternation renewal process. Wang \& Pham [14] discussed the availability considering imperfect repair using quasi-renewal theory. In the literatures, most of the researches on imperfect maintenance and replacement models assume underlying failure time distributions. However, most working systems deteriorate continuously due to usage or age. Deterioration is a process where important parameters of the system gradually worsen and if left unattended, the process leads to deterioration failure. In order to properly model the temporal variability of deterioration, the researchers must rely on the stochastic processes (such as Markov processes). Markov processes include stochastic processes with independent increments like the Brownian motion with drift, the compound Poisson process and the gamma process. For the stochastic modeling of monotonic and gradual deterioration, the gamma process is most appropriate $[15,16]$. According to van Noortwijk [17], Kallen [18], the gamma process is suitable to model gradual damage monotonically accumulating over time in a sequence of tiny increments, such as wear, fatigue, corrosion, crack growth, erosion, consumption, creep, swell, degrading health index, etc.

This paper presents an availability model for stochastic degrading systems under continuous monitoring. Impact of imperfect maintenance actions on the system reliability is investigated. Furthermore, the explicit expression for the availability of the system is evaluated. This model, based on degradation data, could relax the strict assumptions on some failure time distributions. In addition, the method, considering different degradation rules and maintenance policies, should be more reasonable than the method considering the systems as whole.

\section{SYSTEM DESCRIPTION AND ASSUMPTIONS}

We study the availability model for a stochastic deteriorating system by making the following assumptions:

Assumption 1. States of a degrading component leading to failure can be described by a continuous-state stochastic process, $X(t)$, having the initial state $x_{0}=0$. Initially, the two components are both new. If the $X(t)$ of one component exceeds the preventive maintenance (PM) threshold $\xi_{i}$, 
PM is made immediately. If one component is in the period of PM, and the state of other component exceeds the preventive maintenance (PM) threshold $\xi_{i}$, the later one must wait for repair and the system is down.

Assumption 2. The maintenance policy $N$ is applied by which the component is replaced by an identical new one at the $N$ th PM of the component. Assume that the system's component is replaced by a new and identical one, and the replacement time is negligible.

Assumption 3. Repairman would preferentially repair the one where the, $X(t)$, exceeds the PM threshold first. If the state of other one also exceeds the PM threshold, this one would wait for repairs, the waiting time is added in repair time.

Assumption 4. $X_{n}^{(i)}$ denotes the operating time between the $n-1$ th maintenance and $n$th maintenance, and $Y_{n}^{(i)}$ denote the $n$th maintenance time. $X_{n}^{(i)}$ and $Y_{n}^{(i)}$ are mutually independent.

Assumption 5. Assumes that $X(t)$ is a gamma process, and consecutive maintenance time forms a increasing geometrical process.

\section{DEGRADATION CHARACTERISTIC OF SYSTEM}

Deterioration can be regarded as a time-dependent stochastic process $\{X(t), t \geq 0\}$ where $X(t)$ is a random quantity for all $t \geq 0$. In order for the stochastic deterioration process to proceed in one direction, we can best consider it as a gamma process (see, e.g. $[19,20])$. The gamma process is a stochastic process with independent non-negative increments having a gamma distribution with identical scale parameter. We will use the following definition for the gamma density with shape parameter $\alpha>0$ and scale parameter $\beta>0$.

$G a(x \mid \alpha, \beta)=\left(\beta^{\alpha} / \Gamma(\alpha)\right) x^{\alpha-1} \exp (-\beta x) I_{(0, \infty)}(x)$

where $\Gamma(\alpha)=\int_{0}^{\infty} t^{\alpha-1} e^{-t} d t$ is the gamma function and the indicator function is defined as: $I_{(0, \infty)}(x)=1$ for $x \in(0, \infty)$ and zero otherwise. The stationary gamma process with shape function at $\alpha t>0$ and scale parameter $\beta>0$ is a continuous-time process $\{X(t) ; t>0\}$ with the following properties: i) $X(0)=0$ with probability one,

ii) $\Delta X(t)=X(t+\Delta t)-X(t) \sim G a(\alpha \Delta t, \beta)$ for $t \geq 0, \Delta t>0$, iii) $X(t)$ has independent increments.

Component is said to fail when its state, $X(t)$, crosses the threshold $\zeta_{i}$. Let the time at which failure occurs be denoted by the lifetime $T$. Due to the gamma distributed deterioration, Eq. (1), the lifetime distribution can then be written as:

$$
\begin{gathered}
F_{T}(t)=\operatorname{Pr}(T \leq t) \\
=\operatorname{Pr}\left(X(t) \geq \zeta_{i}\right)=\int_{\zeta_{i}}^{\infty} f_{X(t)}(x) d x=\frac{\Gamma\left(v(t), \zeta_{i} \beta\right)}{\Gamma(v(t))}
\end{gathered}
$$

where, $\Gamma(\omega, z)$ denotes the incomplete gamma function, defined as $\Gamma(\omega, z)=\int_{\omega}^{\infty} u^{z-1} e^{-u} d u$.

Given the increments $\left(\Delta x_{i}, \Delta t_{i}\right), i=1,2, \cdots, n$ of a gamma process $X(t)$, the likelihood functions for $\alpha$ and $\beta$ can be easily established. Recall from the definition of the gamma process,

$$
\Delta X_{i} \sim G_{a}\left(\alpha \Delta t_{i}, \beta\right)=\frac{\left(\Delta x_{i} / \beta\right)^{\alpha \Delta t_{i}-1}}{\beta \Gamma\left(\alpha \Delta t_{i}\right)} \exp \left(-\Delta x_{i} / \beta\right) .
$$

The log likelihood function is

$\ln L(\alpha, \beta)=\sum_{i=1}^{n}\left(\alpha \Delta t_{i}-1\right) \ln \Delta x_{i}-\alpha t_{n} \ln \beta-\sum_{i=1}^{n} \ln \Gamma\left(\alpha \Delta t_{i}\right)-\frac{x_{n}}{\beta}$.

We can estimate the two parameters by maximum likelihood estimates (MLE) method.

In general, the expectation of the $i$ st inter-maintenance time, $X\left(t_{n}^{*}\right)$, for a given $\xi_{i}$ is

$$
E\left[X_{n}^{(i)}\right]=E\left[E\left[X_{n}^{(i)} \mid X\left(t_{n-1}^{*}\right)\right]\right]=\int_{0}^{\xi_{i}} E\left[X_{n}^{(i)} \mid X\left(t_{n-1}^{*}\right)\right] f_{X\left(t_{n-1}^{*}\right)}(x) d x
$$

where, $f_{X\left(t_{n}^{*}\right)}(x)$ is the pdf $X\left(t_{n}^{*}\right)$, the state of a degrading system immediately after the $n$th imperfect maintenance action. $X\left(t_{n}^{*}\right)$ is a random variable, and its distribution is constrained to a finite interval, $\left[0, \xi_{i}\right]$. Such quantity relies on the degree of maintenance, which will at least bring the system back to a better state than the maintenance threshold. Under the gamma process model, the unconditional expectation of inter-maintenance time, $X\left(t_{n}^{*}\right)$, can be expressed as [21].

$$
E\left[X_{n}^{(i)}\right]=\int_{0}^{\xi_{i}} \int_{0}^{\infty}\left(1-\frac{\Gamma\left(\alpha_{i} t, \beta_{i} \xi_{i}\right)}{\Gamma\left(\alpha_{i} t\right)}\right) f_{X\left(t_{n-1}\right)}(x) d t d x
$$

Let us discuss the distribution of $X\left(t_{n}^{*}\right)$, several probabilistic models could be utilized. Specifically, we employ Beta distribution to depict $X\left(t_{n}^{*}\right)$, such that a series of puf's, $f_{X\left(t_{n}^{*}\right)}(x), i \in[1, N]$, is defined:

$$
f_{X\left(t_{n}^{*}\right)}(x)=\frac{1}{\xi_{i}} \frac{\Gamma\left(p_{n}+q_{n}\right)}{\Gamma\left(p_{n}\right) \Gamma\left(q_{n}\right)}\left(\frac{x}{\xi_{i}}\right)^{p_{n}-1}\left(1-\frac{x}{\xi_{i}}\right)^{q_{n}-1} I_{\left\{0 \leq x \leq \xi_{i}\right\}},
$$

Where, the model parameters $p_{n}>0$ and $q_{n}>0$ can be estimated using the MLE.

According to the definition of geometrical process [3], the distribution of $Y_{n}^{(i)}$ is given by 


$$
G_{n}^{(i)}(t)=G\left(b_{i}^{n-1} t\right)=1-\exp \left(-b_{i}^{n-1} \mu_{i} t\right),
$$

where, $0<b_{i} \leq 1, \mu_{i}>0$ are repair rate and geometrical rate, respectively. Therefore, the expectation of $Y_{n}^{(i)}$ is described by

$$
E\left[Y_{n}^{(i)}\right]=\frac{\mu_{i}}{b_{i}^{n-1}}
$$

\section{SYSTEM AVAILABILITY MODELING}

Our problem is to determine a method of calculating system availability. Let $A(t)$ denotes the system steady availability under the replacement policy $M=\left(N_{1}, N_{2}, \cdots, N_{k}\right)$, $H_{m}^{(i)}$ and $I_{m}^{(i)}$ denotes the working hours and repairing hours between the $m-1$ th replacement and $m$ th replacement, respectively. Let the total time between the $m-1$ th replacement and $m$ th replacement be $\xi_{m}^{(i)}=H_{m}^{(i)}+I_{m}^{(i)}$, obviously, $\left\{\xi_{1}^{(i)}, \xi_{2}^{(i)}, \cdots\right\}$ is a renewal process. Let $H^{(i)}(t)$ and $I^{(i)}(t)$ denote the total operating time and the total repairing time in $(0, t]$ for component $i$, and $n^{(i)}(t)$ denote the replacement times of component $i$ in $(0, t]$. Furthermore, $\phi^{(i)}(t)$ and $\varphi^{(i)}(t)$ are the operating time and the repairing time between the last replacement and $t$. We have the following equations:

$$
\begin{aligned}
& H^{(i)}(t)=H_{1}^{(i)}+H_{2}^{(i)}+\cdots+H_{n^{(i)}(t)}^{(i)}+\phi^{(i)}(t) \\
& I^{(i)}(t)=I_{1}^{(i)}+I_{2}^{(i)}+\cdots+I_{n^{(i)}(t)}^{(i)}+\varphi^{(i)}(t)
\end{aligned}
$$

According to the definitions of $H_{n}^{(i)}$ and $I_{n}^{(i)}$, we have

$$
H_{m}^{(i)}=\sum_{n=1}^{N_{i}} X_{n}^{(i)}, I_{m}^{(i)}=\sum_{n=1}^{N_{i}-1} Y_{n}^{(i)}
$$

in which $m=1,2, \cdots, n^{(i)}(t)$. According to the model assumption, $t=H^{(i)}(t)+\sum_{i=1}^{k} I^{(i)}(t), \quad k \in(0,1, \cdots)$. Therefore, the system steady availability can then be written as

$$
A(t)=\lim _{t \rightarrow \infty} \frac{E H^{(i)}(t)}{E\left[H^{(i)}(t)+\sum_{i=1}^{k} I^{(i)}(t)\right]}=\lim _{t \rightarrow \infty} \frac{1}{1+\sum_{i=1}^{k} \frac{E\left[I^{(i)}(t)\right]}{E\left[H^{(i)}(t)\right]}}
$$

Because $\left\{H_{1}^{(i)}, H_{2}^{(i)}, \cdots\right\},\left\{I_{1}^{(i)}, I_{2}^{(i)}, \cdots\right\}$ are respectively a renewal process $\mathrm{RP}$, then according to the renewal reward theorem (see, for example, Ross [22]), we have

$$
\lim _{t \rightarrow \infty} \frac{E\left[I^{(i)}(t)\right]}{E\left[H^{(i)}(t)\right]}=\frac{\lim _{t \rightarrow \infty} \frac{E\left[I^{(i)}(t)\right]}{t}}{\lim _{t \rightarrow \infty} \frac{E\left[H^{(i)}(t)\right]}{t}}=\frac{\frac{E I_{1}^{(i)}}{E T_{i}}}{\frac{E H_{1}^{(i)}}{E T_{i}}}=\frac{E I_{1}^{(i)}}{E H_{1}^{(i)}}
$$

$\lim _{t \rightarrow \infty} \frac{E\left[n^{(i)}(t)\right]}{E\left[H^{(i)}(t)\right]}=\frac{1}{E H_{1}^{(i)}}$

According to the anterior analysis, we have

$$
\begin{aligned}
& E H_{1}^{(i)}=\sum_{n=1}^{N_{i}} \int_{0}^{\xi_{i}} \int_{0}^{\infty}\left(1-\frac{\Gamma\left(\alpha_{i} t, \beta_{i} \xi_{i}\right)}{\Gamma\left(\alpha_{i} t\right)}\right) f_{X\left(t_{n}^{*}\right)}(x) d t d x \\
& E I_{1}^{(i)}=E\left(\sum_{n=1}^{N_{i}-1} Y_{n}^{(i)}\right)=\sum_{n=1}^{N_{i}-1} \frac{\mu_{i}}{b_{i}^{n-1}}
\end{aligned}
$$

Substituting of the results above in Eq. (11), we have

$$
\begin{aligned}
& A(t)=\frac{1}{1+\sum_{i=1}^{k} \frac{E I_{1}^{(i)}}{E H_{1}^{(i)}}}=\frac{1}{1+\sum_{i=1}^{k}\left[\sum_{n=1}^{N_{i}-1} \frac{\mu_{i}}{b_{i}^{n-1}}\right] / M} \\
& M=\sum_{n=1}^{N_{i}} \int_{0}^{\xi_{i}} \int_{0}^{\infty}\left(1-\frac{\Gamma\left(a_{i} t, \beta_{i} \xi_{i}\right)}{\Gamma\left(a_{i} t\right)}\right) f_{X\left(t_{n}^{*}\right)}(x) d t d x
\end{aligned}
$$

\section{NUMERICAL EXAMPLE}

The water pump which is a main component of engineering equipment is studied for demonstrating the model proposed in this paper. According to the experts of equipment state evaluation, the temperature and the vibration range maybe the best tokens of the state of system. Therefore, we choose the data of temperature and vibration range as the decision-making warranty. The temperature and vibration range should increase with the working time of system, if the temperature or the vibration range value exceeds some threshold, we think electricity engine or the pump, as the failed one.

As shown in Table 1, some data from censorial apparatus is adopted in the numerical example. Using the MLE technique introduced in section 3 , the estimates are $\left(\alpha_{1}=4, \beta_{1}=0.25\right)$ and $\left(\alpha_{2}=5, \beta_{2}=0.3\right)$. As a series repairable system composed of two components, (14) could rewrite as:

$A(t)=A\left(N_{1}, N_{2}\right)=1 /\left[1+\frac{E I_{1}^{(1)}}{E H_{1}^{(1)}}+\frac{E I_{1}^{(2)}}{E H_{1}^{(2)}}\right]$

Let the other parameter values be: $\mu_{1}=\mu_{2}=0.8$, $b_{1}=b_{2}=0.9, \xi_{1}=38, \xi_{2}=58$. Substituting the above values into the expression (15) and overpassing numerical calculation, we can obtain some results presented in Table 2 and Fig. (1). It is easy to find that the system steady availability varies with $\left(N_{1}, N_{2}\right)$ monotone. In other words, the system steady availability decreases with the increase of replacement times. For fixed $N_{1}$, the relations of $A(t)$ and $N_{2}$ is presented in Fig. (2). In contrary, we can find optimal replacement times by setting certain availability from the expression (14). For example, if the minimal availability is 0.98 then the optimal replacement policy is $\left(N_{1}=3, N_{2}=3\right)$ according to Tables $\mathbf{2}$ and $\mathbf{3}$. 
Table 1. Some data of vibration range value and temperature.

\begin{tabular}{|c|c|c|c|c|c|}
\hline Working Days & State Data (mm) & State Data $\left({ }^{\circ} \mathrm{C}\right)$ & Working Days & State Data $(\mathbf{m m})$ & State Data $\left({ }^{\circ} \mathbf{C}\right)$ \\
\hline 1 & 35.026 & 54.002 & 400 & 36.205 & 58.005 \\
\hline 60 & 36.002 & 56.020 & 440 & 36.367 & 56.337 \\
\hline 120 & 36.041 & 57.076 & 480 & 36.775 & 56.549 \\
\hline 180 & 37.138 & 57.237 & 520 & 37.647 & 57.225 \\
\hline 240 & 37.438 & 57.357 & 550 & 38.312 & 57.913 \\
\hline 280 & 37.828 & 57.570 & 580 & 36.833 & 58.007 \\
\hline 320 & 38.155 & 57.798 & 610 & 36.904 & 56.703 \\
\hline 360 & 36.190 & 57.968 & 640 & 37.890 & 57.041 \\
\hline
\end{tabular}

Table 2. The values of availability under $\left(N_{1}, N_{2}\right)$.

\begin{tabular}{|c|c|c|c|c|}
\hline $\mathbf{N 1} \backslash \mathbf{N 2}$ & $\mathbf{1}$ & $\mathbf{2}$ & $\mathbf{3}$ \\
\hline \hline 1 & 0.9821 & 0.9817 & 0.9813 & 0.9808 \\
\hline 2 & 0.9815 & 0.9811 & 0.9807 & $\mathbf{0 . 9 8 0 0}$ \\
\hline 3 & 0.9808 & 0.9804 & 0.9793 & 0.9802 \\
\hline 4 & 0.9801 & 0.9798 & 0.9786 & 0.9782 \\
\hline 5 & 0.9794 & 0.9790 & 0.9778 & 0.9774 \\
\hline 7 & 0.9786 & 0.9782 & 0.9770 & 0.9765 \\
\hline
\end{tabular}

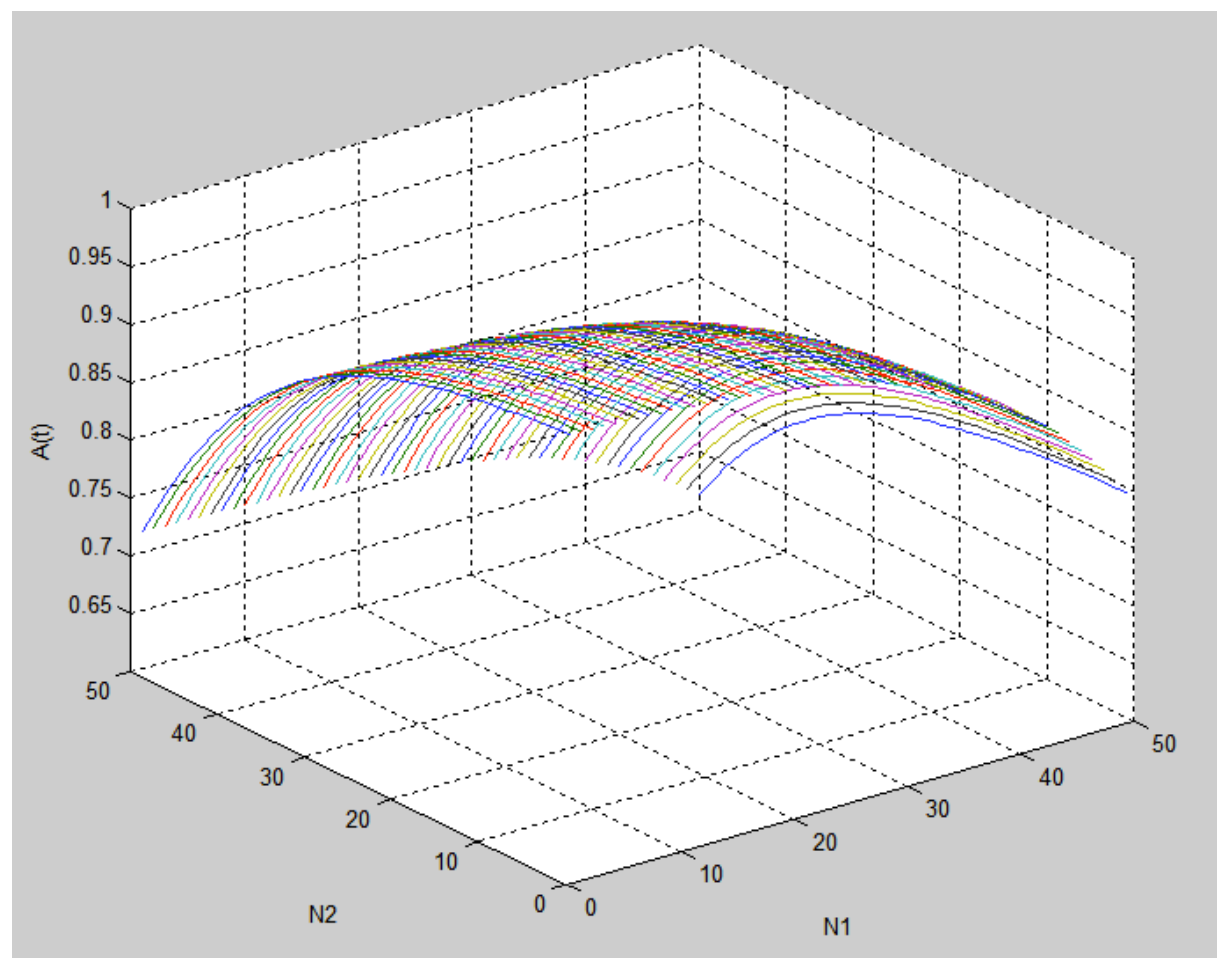

Fig. (1). The plots availability of against $\left(N_{1}, N_{2}\right)$. 


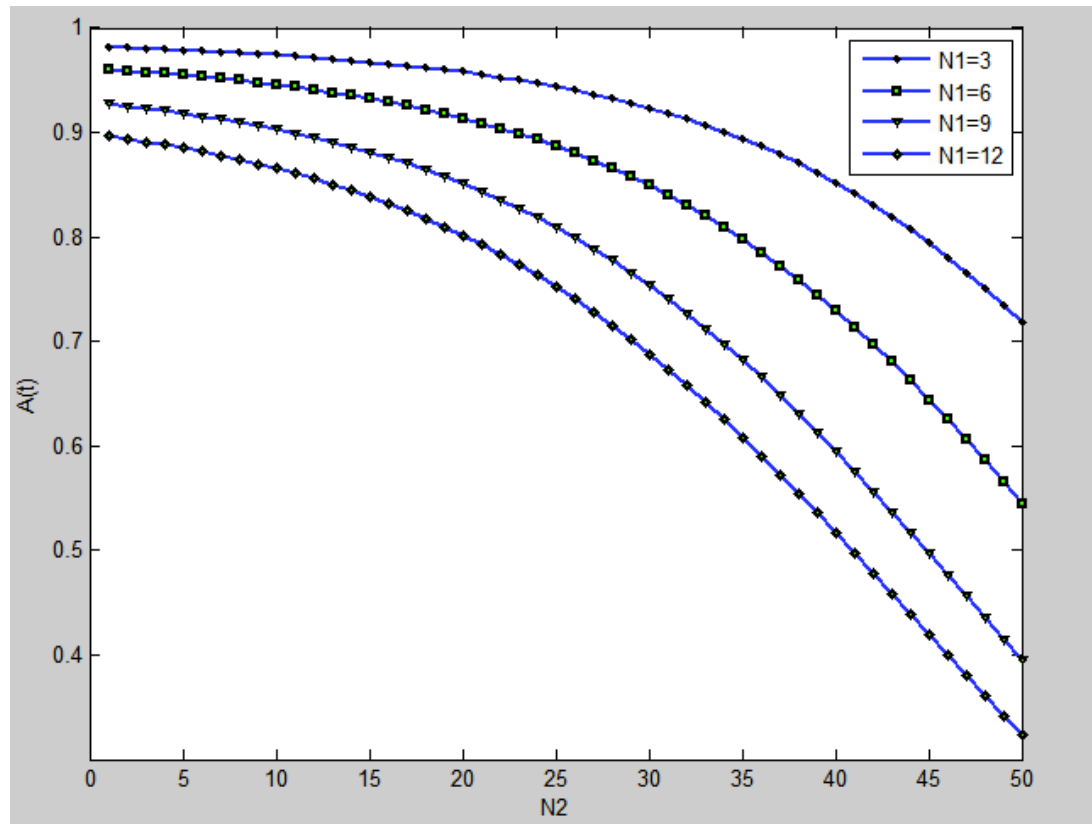

Fig. (2). The plots availability of against $N_{2}$.

Table 3. The values of availability under $\left(N_{1}, N_{2}\right)$.

\begin{tabular}{|c|c|c|c|c|}
\hline $\mathbf{N 1} \mathbf{N 2}$ & $\mathbf{5}$ & $\mathbf{6}$ & $\mathbf{7}$ & $\mathbf{8}$ \\
\hline \hline 1 & 0.9803 & 0.9798 & 0.9793 & 0.9787 \\
\hline 2 & 0.9798 & 0.9792 & 0.9787 & 0.9781 \\
\hline 3 & 0.9791 & 0.9786 & 0.9781 & 0.9775 \\
\hline 4 & 0.9784 & 0.9779 & 0.9774 & 0.9768 \\
\hline 5 & 0.9777 & 0.9772 & 0.9767 & 0.9753 \\
\hline 6 & 0.9769 & 0.9764 & 0.9759 & 0.9750 \\
\hline
\end{tabular}

\section{CONCLUSION}

In this work, we have presented an availability model which takes into account the imperfect maintenance actions. In the literatures, most of the researches consider the system as a whole, however, a method of calculating availability, considering continuous inspection on each component, is proposed in this paper. We utilize a gamma process in order to relax the assumption from the multi-state space to the continuous-state space. The aging properties relevant to the proposed maintenance policy are also addressed. This paper assumes that system after maintenance is not as good as new such that the state of a degrading system immediately after the imperfect maintenance action is assumed as a random variable and the successive maintenance time follows a geometric process. At last, a numerical example for a degrading system model is presented to demonstrate the use of this model in practical applications.

\section{CONFLICT OF INTEREST}

The authors confirm that this article content has no conflict of interest.

\section{ACKNOWLEDGEMENTS}

Declared none.

\section{REFERENCES}

[1] M. Brown, F. Proschan, "Imperfect repair," Application Probability, no. 20, pp. 851-859, 1983.

[2] H. Pham, and H. Wang, "Imperfect maintenance," European Journal of Operational Research, vol. 94, no. 3, pp. 425-438, 1996.

[3] Y. Lam, "A note on the optimal replacement problem," Advances in Applied Probability, vol. 20, no. 2, pp. 479-482, 1988.

[4] Lam Y, "A geometric process maintenance model with preventive repair," European Journal of Operational Research, vol. 182, no. 2, pp. 806-819, 2007.

[5] Y. L. Zhang, G. J. Wang, "A bivariate optimal replacement policy for a multistate repairable system," Reliability Engineering and System Safety, vol. 92, pp. 535-542, 2007.

[6] G. J. Wang, and Y. L. Zhang, "An optimal replacement policy for a two-component series system assuming geometric process repair," Computers and Mathematics with Applications, vol. 54, pp. 192202, 2007.

[7] M. Kijima, "Some results for repairable system with general repair," Journal of Applied Probability, vol. 26, pp. 89-102, 1989.

[8] S. Martorell, A. Sanchez, and V. Serradell, "Age-dependent reliability model considering effects of maintenance and working conditions," Reliability Engineering and System Safety, vol. 64, pp. 19-31, 1999. 
[9] S. H. Sheu, R. H. Yeh, Y. Lin, and M. Juang, "A Bayesian perspective on age replacement with minimal repair," Reliability Engineering and System Safety, vol. 65, pp. 55-64, 1999.

[10] J. H. Chiang, J. Yuan, "Optimal maintenance policy for a Markovian system under periodic inspection," Reliability Engineering and System Safety, vol. 71, pp. 165-172, 2001.

[11] R. Jiang, P. Ji, "Age replacement policy: a multi-attribute value model," Reliability Engineering and System Safety, vol. 76, pp. 311-318, 2002

[12] S. Lyer, "Availability results for imperfect repair," Sankhya: the Indian Journal of Statistics, vol. 54, no. 2, pp. 249-259, 1992.

[13] M. Zhao, "Availability for repairable components and series system," IEEE Transaction Reliability, vol. 43, no. 2, pp. 329-334, 1994.

[14] H. Wang, and H. Pham, "Availability and maintenance of series systems subject to imperfect repair and correlated failure and repair," European Journal of Operational Research, vol. 174, pp. 1706-1722, 2006.

[15] A. Grall, C. Berenguer, and L. Dieulle, "A condition-based maintenance policy for stochastically deteriorating systems," Reliability Engineering and System Safety, vol. 76, pp. 167-180, 2002.
[16] S. Bloch-Mercier, "A preventive maintenance policy with sequential checking procedure for a Markov deteriorating system," European Journal of Operational Research, vol. 147, pp. 548-576, 2002.

[17] J. M. van Noortwijk, J. A. M. van der Weide, M.J. Kallena, and M.D. Pandey, " Gamma processes and peaks-over-threshold distributions for time-dependent reliability," Reliability Engineering and System Safety, vol. 92, no. 12, pp. 1651-1658, 2007.

[18] M. J. Kallen, and J. M.van Noortwijk, "Optimal maintenance decisions under imperfect inspection," Reliability Engineering and System Safety, vol. 90, pp. 177-185, 2005.

[19] J. M. van Noortwijk, and D. M. Frangopol, "Two probabilistic lifecycle maintenance models for deteriorating civil infrastructures," Probabilistic Engineering Mechanics, vol. 19, no. 4, pp. 345-359, 2004.

[20] L. Tan, and S. Gong, and B. Guo, "Risk-based Inspection optimization of the deteriorating system," Progress in Safety Science and Technology, pp. 1874-1878, 2008.

[21] H. T. Liao, A. Elsayed, and L. Y. Chan, "Maintenance of continuously monitored degrading systems," European Journal of Operational Research, vol. 175, pp. 821-835, 2006.

[22] S. M. Ross, Stochastic Process, $2^{\text {nd }}$ ed. Wiley, New York, 1999.

(C) Chun et al.; Licensee Bentham Open.

This is an open access article licensed under the terms of the Creative Commons Attribution Non-Commercial License (http://creativecommons.org/licenses/by-nc/3.0/) which permits unrestricted, non-commercial use, distribution and reproduction in any medium, provided the work is properly cited. 